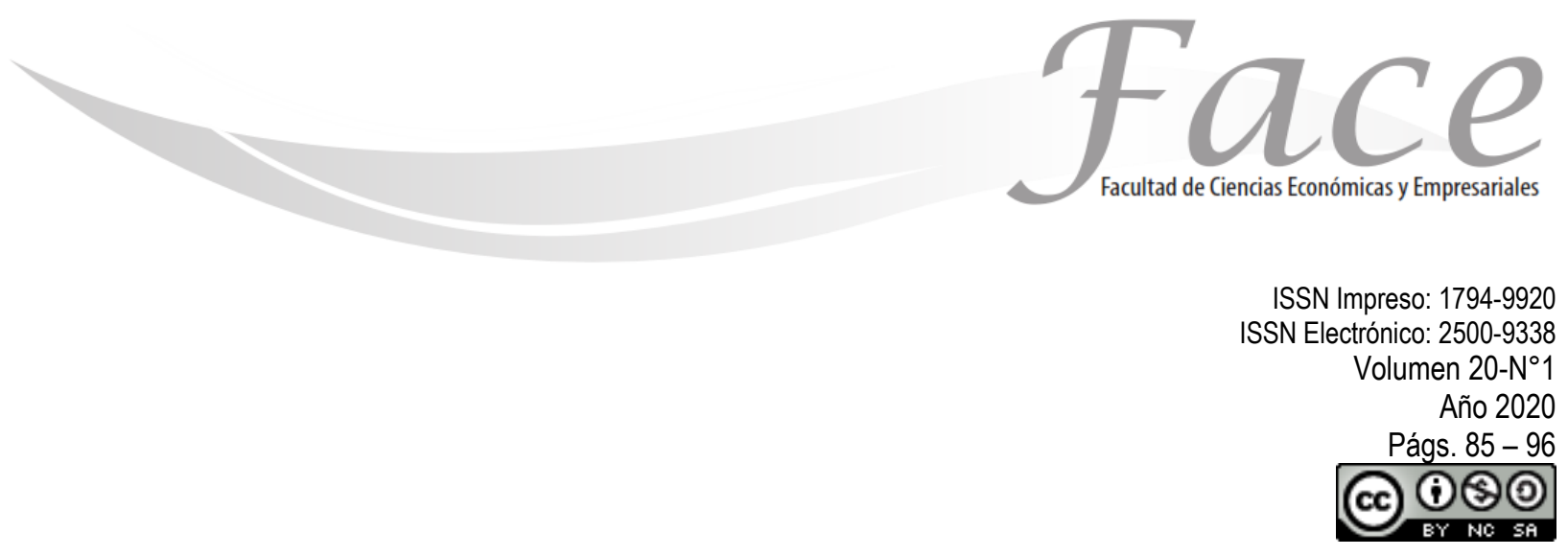

\title{
LAS ACTITUDES MEDIOAMBIENTALES DE LOS CONSUMIDORES: ANÁLISIS COMPARATIVO EN LAS CIUDADES DE SAN LUIS DE POTOSÍ, MÉXICO Y MEDELLÍN COLOMBIA *
}

\author{
Mónica Eugenia Peñalosa Otero ** \\ Enlace ORCID: http://orcid.org/0000-0002-2208-9224 \\ Diana María López Celis *** \\ Enlace ORCID: http://orcid.org/0000-0002-7949-0625 \\ Marco Francisco Martínez Aguilar **** \\ Enlace ORCID: https://orcid.org/0000-0002-3993-2513
}

Fecha de Recepción: Abril 2 de 2020

Fecha de Aprobación: Julio 1 de 2020

\section{Resumen:}

A lo largo de este trabajo, se presentan los resultados de la investigación realizada entre investigadores de la Universidad Jorge Tadeo Lozano en Colombia y la Universidad Autónoma de San Luis de Potosí en México, por medio de la cual se busca hacer un comparativo de las actitudes medioambientales de los en las ciudades de Medellín, Colombia y San Luis de Potosí, México.

Entre los hallazgos cabe destacar que se encontró una actitud o predisposición aprendida positiva, respecto a los temas medioambientales tanto para los consumidores de San Luis de Potosí como para Medellín Colombia.

Palabras clave: ambiente, actitud ambiental, consumo responsable.

\footnotetext{
* Artículo de reflexión resultado de la investigación titulada "Tipos de consumidores de acuerdo con la actitud ambiental: análisis comparativo entre San Luis De Potosi-México y Medellín-Colombia.

${ }^{* *}$ Profesora Asociada de la Facultad de Ciencias Económicas y Administrativas, Universidad Jorge Tadeo Lozano, Bogotá, Colombia. Magister en Mercadeo Agroindustrial. Profesional en Relaciones Internacionales. Investigadora Jr. de Colciencias. Se ha venido desempeñando en los últimos años como Docente Investigadora en la Universidad Jorge Tadeo Lozano, la Universidad de La Salle y la Universidad de Pamplona - Colombia. Correo electrónico: monica.penalosa@utadeo.edu.co

*** Profesora Asociada de la Facultad de Ciencias Económicas y Administrativas, Universidad Jorge Tadeo Lozano, Bogotá, Colombia. Magister en Psicología del Consumidor, especialista en Psicología del Consumidor. Profesional en Psicología. Investigadora Jr. de Colciencias. Se ha venido desempeñando en los últimos años como Docente - Investigadora en la Universidad Jorge Tadeo Lozano, la Universidad Central y la Universidad Javeriana- Colombia. Correo electrónico: diana.lopez@utadeo.edu.co

**** Maestro en educación adscrito a la Coordinación Académica Región Altiplano. Universidad Autónoma de San Luis Potosí. México. Correo electrónico: marcos.martinez@uaslp.mx
} 


\title{
THE ENVIRONMENTAL ATTITUDES OF CONSUMERS: COMPARATIVE ANALYSIS IN THE CITIES OF SAN LUIS DE POTOSÍ, MEXICO AND MEDELLÍN COLOMBIA
}

\begin{abstract}
:
Throughout this work, we present the results of the research carried out by researchers from the Universidad Jorge Tadeo Lozano in Colombia and the Universidad Autónoma de San Luis de Potosí in Mexico, through which we seek to make a comparison of environmental attitudes in the cities of Medellín, Colombia and San Luis de Potosí, Mexico.

Among the findings, it is worth mentioning that a positive learned attitude or predisposition was found, regarding environmental issues for both consumers in San Luis de Potosi and Medellin Colombia.
\end{abstract}

Keywords: environment, environmental attitude, responsible consumption

\section{AS ATITUDES AMBIENTAIS DOS CONSUMIDORES: ANÁLISE COMPARATIVA NAS CIDADES DE SAN LUIS DE POTOSÍ, MÉXICO E MEDELLÍN COLÔMBIA}

Resumo:

Ao longo deste trabalho, são apresentados os resultados da pesquisa realizada entre pesquisadores da Universidade Jorge Tadeo Lozano, na Colômbia, e da Universidade Autônoma de San Luis de Potosí, no México, por meio da qual se busca uma comparação de atitudes. condições ambientais nas cidades de Medellín, Colômbia e San Luis de Potosí, México.

Entre os achados, deve-se destacar que foi encontrada uma atitude ou predisposição positiva aprendida, em relação às questões ambientais, tanto para os consumidores em San Luis de Potosí quanto em Medellín, Colômbia.

Palavras-chave: meio ambiente, atitude ambiental, consumo responsável. 


\section{INTRODUCCIÓN:}

Los cambios sociales y culturales, sumado al incremento en las tecnologías, han modificado algunos comportamientos del ser humano, sin dejar de lado, las implicaciones que estos ocasionan desde una perspectiva medioambiental, dado que, cada vez es mayor la preocupación frente a la problemática del consumo desmesurado de nuestros recursos. Por tanto, desde la academia se ha despertado la necesidad de investigar temas ligados al consumo y a la actitud de ser humano frente al entorno, con la finalidad de conocer cuáles son las actitudes, los hábitos de compra y los factores determinantes de consumo desde un ámbito ambiental.

Por tanto, podría llegar a pensarse que los consumidores expresan su inquietud por el ambiente y son motivados por necesidades universales $\mathrm{y} / \mathrm{o}$ colectivas, lo que a su vez, genera que grupos poblacionales manifiesten esa preocupación a través de su consumo y como consecuencia, las organizaciones comiencen a considerar abastecer a un mercado que demande productos respetuosos con la naturaleza (Vargas Martínez, López Moreda, \& Martínez Cervantes, 2014).

Desde el mercadeo es indispensable poder encontrar grupos con características similares para realizar estrategias diferenciadas, con el interés de valorar el atractivo de cada segmento (Kotler \& Armstrong, Fundamentos de marketing, 2013).

Por lo anterior, esta investigación pretende aportar evidencia para diferenciar los tipos de consumidores de acuerdo con su actitud ambiental, y con ello, poder discriminar las similitudes 0 diferencias en las poblaciones de San Luis de Potosí en México y Medellín en Colombia, dado que estas dos ciudades son consideradas como similares, al ser intermedias y destacarse porque gran parte de su economía se basa en el sector industrial.
Se desarrolló una investigación concluyente descriptiva durante el segundo semestre de 2019, con una muestra de 385 sujetos para cada ciudad, con una confiabilidad del $95 \%$ y margen de error del $5 \%$; donde se pudo determinar que tanto en Medellín (Colombia) como San Luis de Potosí (México), los consumidores de manera general tienen una actitud positiva frente a temas medioambientales, la cual se ve reflejada en su actuar; sin embargo, al evaluar en específico la conducta de consumo en San Luis de Potosí, se evidencia que no tienen realmente prácticas puntuales que los identifique tal como sí ocurre con los consumidores de Medellín.

\section{REVISIÓN DE LA LITERATURA}

Durante el siglo $\mathrm{XX}$ en las diversas agendas gubernamentales se pudo observar una creciente preocupación mundial por el agotamiento de los recursos naturales, en su mayoría por el uso irracional que el ser humano les da.

Lo anterior, produjo una serie de convenios y en especial un programa de acciones para el siglo XXI, marcando un hito a nivel global por el reconocimiento de la necesidad de la reconciliación entre la conservación ambiental y el desarrollo económico, mediante la popularización del concepto de desarrollo sostenible. (ONU, 1992).

Sin embargo y a pesar de las propuestas constituidas en las agendas globales y de la inclusión de temas medio ambientales en los planes y programas gubernamentales en cada uno de los países firmantes, los problemas del medio ambiente y el deterioro de los ecosistemas continúan avanzando de forma acelerada. 
La crisis ecológica hace referencia a una conducta mal adaptada; donde las ciencias de la conducta adquieren la responsabilidad de entender, predecir y modificar aquellas conductas de la población más implicadas en el desarrollo de esta problemática. (González Maloney \&Ward, 1973).

\section{Actitud ambiental}

Según Newhouse (1990), el análisis psicológico de los problemas del medio ambiente basado en las actitudes ha estado muy condicionado por los repetidos intentos de contemplar la posible existencia de una actitud general hacia el medio ambiente o constructo de preocupación ambiental, que pueda explicar la conducta que los seres humanos despliegan con respecto a su medio ambiente. El concepto de actitud, por tanto, ha sido considerado de vital importancia para poder explicar los determinantes de la conducta ecológica 0 ambiental y la forma en que ésta podría ser cambiada en una determinada dirección. Respecto a la psicología ambiental, el Colegio Colombiano de Psicólogos - Colpsic, advierte que esta estudia las variables ambientales de percepción y actitud, permitiendo una mirada complejizante del ser humano y el medio en el cual se desarrolla.

Mientras que Roth (2000), asegura que la psicología ambiental analiza la relación entre el tema ambiental y el comportamiento humano a partir de dos enfoques: la variable ambiental como determinante del comportamiento y los efectos que tiene la conducta en el medio ambiente.

En este sentido y de acuerdo con estos planteamientos, la psicología ambiental como rama de la ciencia, permite la comprensión de la relación entre individuo y medio ambiente, desde una perspectiva conductual; esto implica analizar, cómo el medio ambiente modifica la conducta del individuo y cómo éste a su vez genera una afectación en el medio ambiente, es decir, en el espacio físico, social y territorial que ocupa.

Ahora bien, frente a dicho estudio de la relación entre la conducta del individuo y el medio ambiente, la conducta y la actitud cobran gran relevancia. Para efectos de la presente investigación el concepto de actitud será descrito con mayor profundidad. Las definiciones existentes sobre el concepto de actitud comparten como elementos claves, el carácter de conjunto de convicciones o creencias estables y la predisposición favorable o no para actuar frente a una situación en particular. (González López, 2002).

Holahan (2005), menciona que la actitud ambiental hace referencia a los sentimientos favorables 0 desfavorables que se tiene hacia un problema relacionado con el ambiente natural, por tanto, plantea que la psicología ambiental se ha interesado cada vez más en evaluar las actitudes de las personas con respecto a la conservación del ambiente en la medida en que ha aumentado la conciencia social sobre la necesidad de preservar los recursos naturales. inalmente, hace hincapié en los beneficios que trae consigo el análisis de la relación entre los individuos y el medio ambiente desde la actitud ambiental.

\section{Consumo Responsable}

En la década de 1970, el concepto de consumo verde fue referencia para interrelacionar diferentes disciplinas, entre ellas, la ecológica y el mercadeo, lo cual permitió el desarrollo de investigación en problemáticas ambientales, de esta manera surgen nuevos conceptos como consumo sostenible 0 responsable (Dueñas, Perdomo-Ortiz y Villa, 2014, citados por Pascual et al., 2016). Esta definición describe originalmente lo postulado por El-kington y Hailes (1989), para validar que toda acción del ser humano en las decisiones de consumo puede generar riesgos en la salud, no solo del consumidor, sino de otras especies: "aquellos que causan daño significativo al medio ambiente durante la manufactura, su uso o desperdicio [...]; así como aquellos que implican un maltrato innecesario de animales o que de manera adversa afectan a otros países" (citado por Pascual et al., 2015, p. 75). Esta situación permite incorporar elementos éticos y morales en la conducta del consumidor, en la que satisface una necesidad, pero va más allá de una condición de mercado para analizar condiciones fuera de este, desde el punto de vista del activismo medioambiental, como consciente de una realidad en crisis ambiental. En este sentido, tanto para Shaw y Shiu (2002) y Dueñas et al. (2014), la decisión del consumidor ético se convierte en un elemento de transformación e impacto en la sociedad que depende de la conservación de los recursos naturales, pasando 
de un concepto antiguo de solo producción a un escenario de comportamientos colectivos para configurar un concepto más integral en un consumo socialmente responsable (CSR) (Pascual et al., 2015).

La formación de una actitud ambiental, sustentada en las prácticas cotidianas, en la importancia que el individuo construye frente al tema ambiental y las relaciones con los productos que consume, entre otros; tiene que ver también con otra categoría de análisis referida al consumo responsable; esta, que tiene estrecha relación con el consumo sostenible, parte de entender las prácticas de consumo desde una variable de ser conscientes frente a lo que se compra y lo que se consume, desde el análisis casi cotidiano y aprehendido de los impactos de ello sobre el medio ambiente.

San Luis de Potosí México y Medellín Colombia como ciudades industriales y los estudios realizados sobre las actitudes respecto al medio ambiente. La revisión de la literatura previa no solo ofrece sólidas evidencias en torno a la importancia de evaluar la actitud de los consumidores acerca del medio ambiente; como parte del consumo responsable, es además hoy en día una obligación ética y una tendencia en los países desarrollados (Webb et al., 2008).

San Luis de Potosí en México y Medellín en Colombia, son dos ciudades que pertenecen a la cultura Latinoamericana, con un auge a nivel industrial. San Luis Potosí se ha convertido en polo mundial tras la inercia de importantes proyectos e inversiones que han elegido a esta ciudad como sede de sus centros de fabricación y operaciones. De otro lado, se presume que el crecimiento del sector industrial es integral, pues, aunque la fortaleza se concentra en la zona metropolitana, se impulsa la capacidad industrial de las cuatro zonas del estado y municipios vecinos a la capital. Ante la llegada de la armadora BMW, la llantera Goodyear y constantes expansiones de importantes firmas; al igual que Medellín, considerada como una ciudad que sobresale como uno de los principales centros financieros, industriales, comerciales y de servicios de Colombia, en especial en los sectores textil, confecciones, metalmecánico, eléctrico y electrónico, telecomunicaciones, automotriz, alimentos y salud, considerados como uno de los principales focos de industrialización en Colombia. Por esta razón, desde la alcaldía de Medellín, se lleva a cabo todo un plan ambiental encaminado a mantener una ciudad sostenible; donde también se involucra a la empresa privada.

Según Mejía (2018), las familias en Medellín no consideran que existen unas condiciones favorables para mantener prácticas de consumo y desecho responsable, dado que para ellos es poca la capacidad del consumidor para generar un impacto real y positivo en el ambiente.

Así mismo, se cree que quienes mantienen prácticas favorables de consumo responsable, son personas cuya educación es alta en términos del hogar, basada en valores bien estructurados y con niveles académicos universitarios superiores que la media. En otras palabras, el entorno social y cultural permea en la conducta del individuo con respecto al medio ambiente.

En Colombia, la ciudad de Medellín se ha caracterizado por tener una actitud positiva con respecto al consumo responsable, lo que ha permitido al consumidor realizar compras con más sentido de responsabilidad, en cuanto al impacto que esta conducta puede tener, no solo para él, sino para su entorno. (Peñalosa Otero \& López Celis, 2018).

Por lo anterior, se evidencia que en el caso colombiano se han desarrollado investigaciones con respecto al tema en mención, sin embargo, en San Luis de Potosí, aún no se encuentra referenciado algún estudio donde se evalúen las actitudes ambientales de los consumidores, siendo una ciudad con un amplio desarrollo industrial, lo cual hace que cobre gran relevancia este estudio, dada su temática y el impacto a nivel del conocimiento de la actitud de los consumidores frente al tema medio ambiental en esta zona de México. 


\section{METODOLOGÍA:}

Esta investigación estuvo a cargo de la Universidad Jorge Tadeo Lozano de Colombia y la Universidad Autónoma de San Luis de Potosí de México, de la mano del acuerdo colaborativo celebrado entre ambas instituciones a partir de julio de 2019 teniendo como objetivo determinar la actitud ambiental de los consumidores en la ciudad de Medellín, Colombia y San Luis de Potosí, México.

La investigación tuvo un enfoque cuantitativo con alcance descriptivo a través de encuestas estructuradas a consumidores de Medellín y San Luis de Potosí mayores de 18 años, que hubieran realizado compras en hipermercados, supermercados, minimercados o tiendas durante el último mes. El estudio tuvo una confiabilidad del $95 \%(n=385)$ y un error muestral del $5 \%$, a través de muestreo no probabilístico.

\section{Instrumento}

La revisión de la literatura llevó a definir y obtener las variables utilizadas, las cuales fueron consideradas como una adaptación al instrumento propuesto por Celedonio Castanedo (1995) sobre la valoración de las cualidades a favor del medio ambiente (EAPA), la cual ha sido validada mediante el análisis factorial y es confiable de acuerdo con el indicador de Alpha de Cronbach. Las variables están redactadas para acordar la etapa actual sobre las actitudes ambientales en el ámbito del social y calcular como son observadas las personas a través del contacto ambiental (Celedonio Castanedo, 1995, p. 258).
En total la escala contiene 17 ítems, de esta escala fueron tomados 9 items que de acuerdo a un análisis preliminar, tienen un mejor desempeño en la medición de las actitudes ambientales considerando cada una de las culturas, y divididos en categorias a juicio de los investigadores, para poderle dar una mejor interpretación de acuerdo al objetivo de investigación. Las escalas utilizadas fueron conservadas y son del tipo Likert, de modo que para cada encuestado existan distintas alternativas graduadas en intensidad de 4 niveles (Alaminos Chica \& Castejón Costa, 2006) donde; muy de acuerdo $=1$, de acuerdo $=2$, en desacuerdo $=3$ y muy en desacuerdo $=4$.

\section{RESULTADOS:}

\section{Análisis Descriptivo}

Para iniciar el análisis es importante conocer en dónde realizan los consumidores las compras habituales del hogar, para lo cual se encontró que tanto en los consumidores de San Luis de Potosí como de Medellín, tienen mayor tendencia a realizar dichas compras en grandes superficies o supermercados de cadena con un $81 \%$ y $78 \%$ respectivamente, sin embargo, se evidenció que los consumidores de Medellín alternan las compras en mini mercados con un $43 \%$ y tiendas de barrio 37\%; mientras que en San Luis de Potosí, la participación de otros establecimientos se encuentra por debajo del $10 \%$.

En el momento de realizarlas compras definitivamente las variables de precio y calidad son las que más se destacan; mientras que las variables eco amigables tienen más baja participación, siendo más importante esta variable en Medellín con un $23 \%$, frente a un $7 \%$ en San Luis de Potosí. 


\section{Gráfica 1. Variables influyentes al momento de la compra}

\begin{tabular}{|c|c|}
\hline MEDELLIN - COLOMBIA & SAN LUIS DE POTOSI - MEXICO \\
\hline $\begin{array}{l}\text { 2. A la hora de comprar un producto } \\
\text { para el consumo del hogar o de } \\
\text { consumo personal, ¿en cuál de los } \\
\text { siguientes aspectos se fija usted? }\end{array}$ & $\begin{array}{l}\text { 2. A la hora de comprar un producto } \\
\text { para el consumo del hogar o de } \\
\text { consumo personal, ¿en cuál de los } \\
\text { siguientes aspectos se fija usted? }\end{array}$ \\
\hline $67 \%$ & \\
\hline Marca $\quad 47 \%$ & Calidad comprobada \\
\hline Calidad $\quad 339 \%$ & Precio del producto $\quad 22 \%$ \\
\hline Precio $\quad 36 \%$ & Marca conocida $\quad 15 \%$ \\
\hline Costumbre $\quad 28 \%$ & Envase $\quad 8 \%$ \\
\hline Producto eco-amıgable $23 \%$ & Producto ecoamigable $\quad \mathbf{7 \%}$ \\
\hline Envase/empaque... $12 \%$ & Relación calidad/precio $\quad 3 \%$ \\
\hline Envase/empaque $\quad \mathbf{8 \%}$ & Otro $=3 \%$ \\
\hline
\end{tabular}

Fuente: Elaboración propia.

Sumada a esta conducta, los consumidores de Medellín prefieren empacar sus compras en la bolsa de tela que acostumbran a llevar en un $72 \%$; mientras que, en San Luis de Potosí, prefieren utilizar bolsa de plástico en un $78 \%$.

Gráfica 2. Cómo empacan las compras

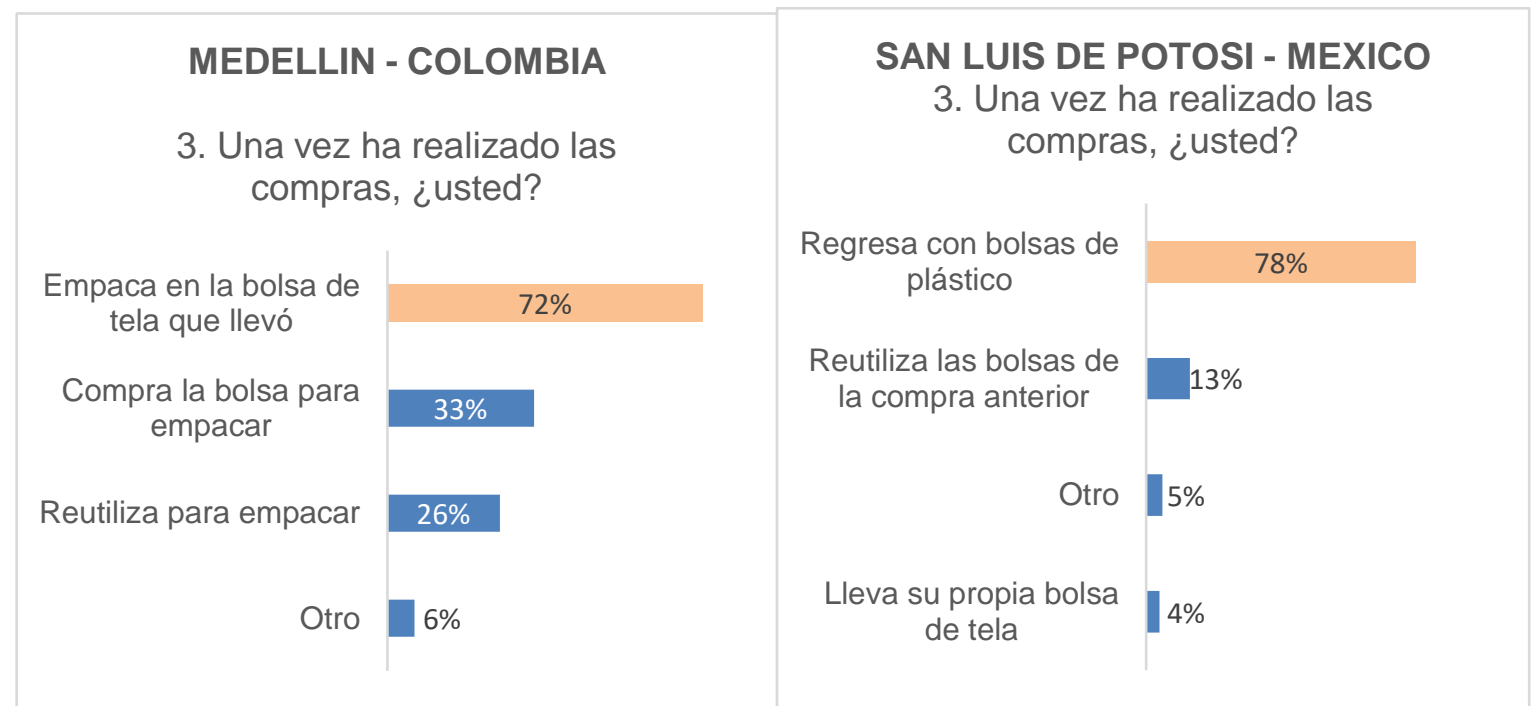


Para los consumidores, resulta importante la migración de los empaques y envases tradicionales a una forma más amigable con el medioambiente; y de ser necesario, apoyan el uso de herramientas por parte del gobierno, en cuanto a normas como por ejemplo los impuestos tanto a nivel individual como a las empresas con el objetivo de generar una mayor conciencia sobre el impacto de sus conductas sobre el medioambiente.

Gráfica 3. Actitud frente a estrategias para reducir el impacto medioambiental negativo

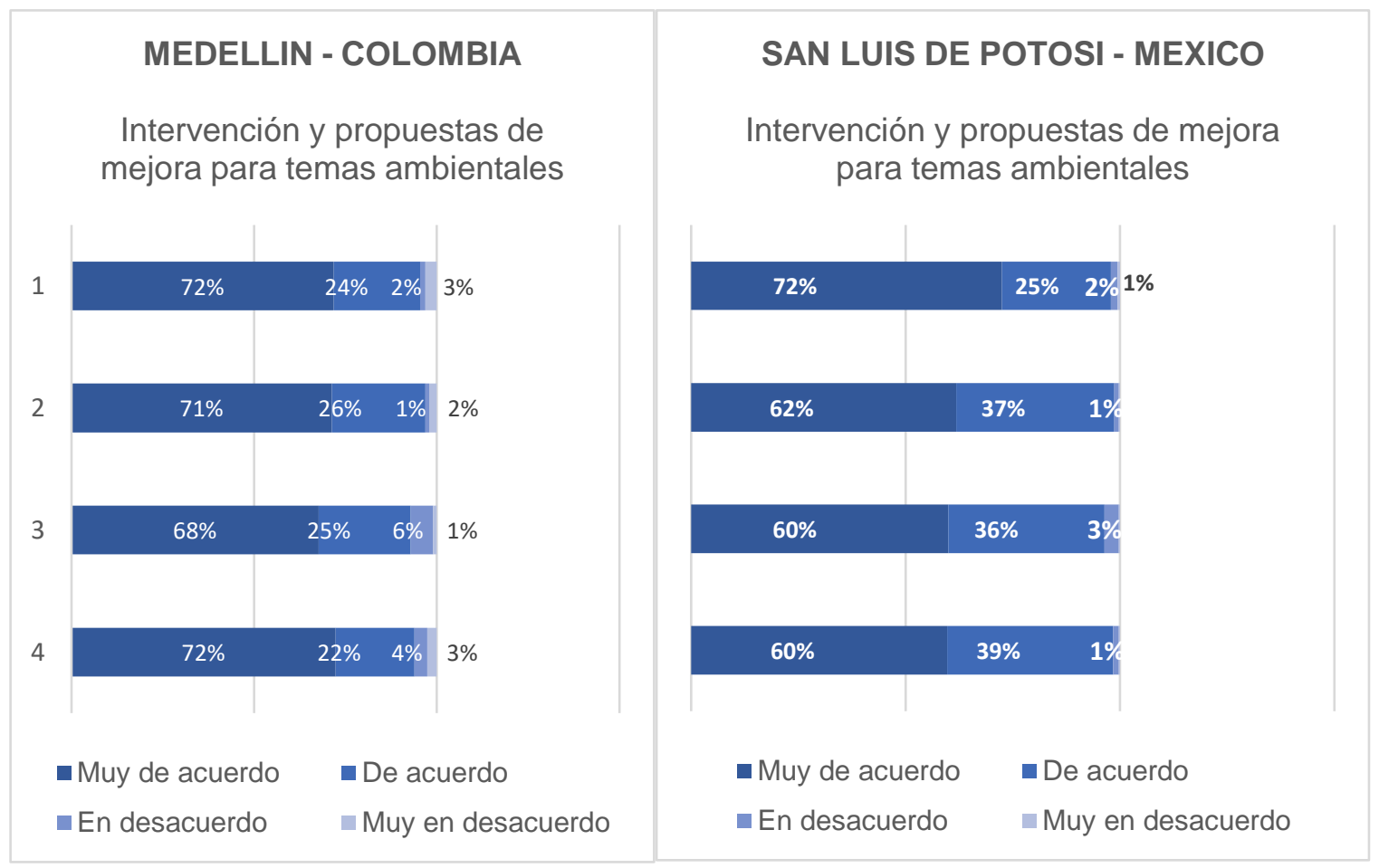

Fuente: Elaboración propia.

\begin{tabular}{ll}
\hline $\mathbf{1}$ & $\begin{array}{l}\text { Apoyo que cobren impuestos a todas aquellas (industrias, empresas o personas) que originen } \\
\text { contaminación. }\end{array}$ \\
\hline $\mathbf{2}$ & $\begin{array}{l}\text { El gobierno debería introducir medidas severas para frenar la contaminación ambiental, ya que poca gente la } \\
\text { regulará por sí misma. }\end{array}$ \\
\hline 3 & $\begin{array}{l}\text { Los productos envasados, deberían de ser de vidrio retornable para evitar la acumulación en el ambiente de } \\
\text { latas y botellas. }\end{array}$ \\
\hline 4 & Debería controlarse el uso y el abuso de las bolsas de plástico. \\
\hline
\end{tabular}


LAS ACTITUDES MEDIOAMBIENTALES DE LOS CONSUMIDORES: ANÁLISIS COMPARATIVO EN LAS CIUDADES DE SAN LUIS DE POTOSÍ, MÉXICO Y MEDELLÍN COLOMBIA

Mónica Eugenia Peñalosa Otero - Diana María López Celis - Marco Francisco Martínez Aguilar

Tanto en los consumidores de Medellín como de San Luis de Potosí, se observa una actitud favorable respecto a tomar un papel activo frente a los temas ambientales; es decir en querer jugar un papel activo en el tema y no simplemente de observadores, al punto de considerar que es un factor que afecta sus vidas a nivel personal y en la sociedad.

Gráfica 4. Actitud adoptada por los consumidores frente al impacto de los temas ambientales.

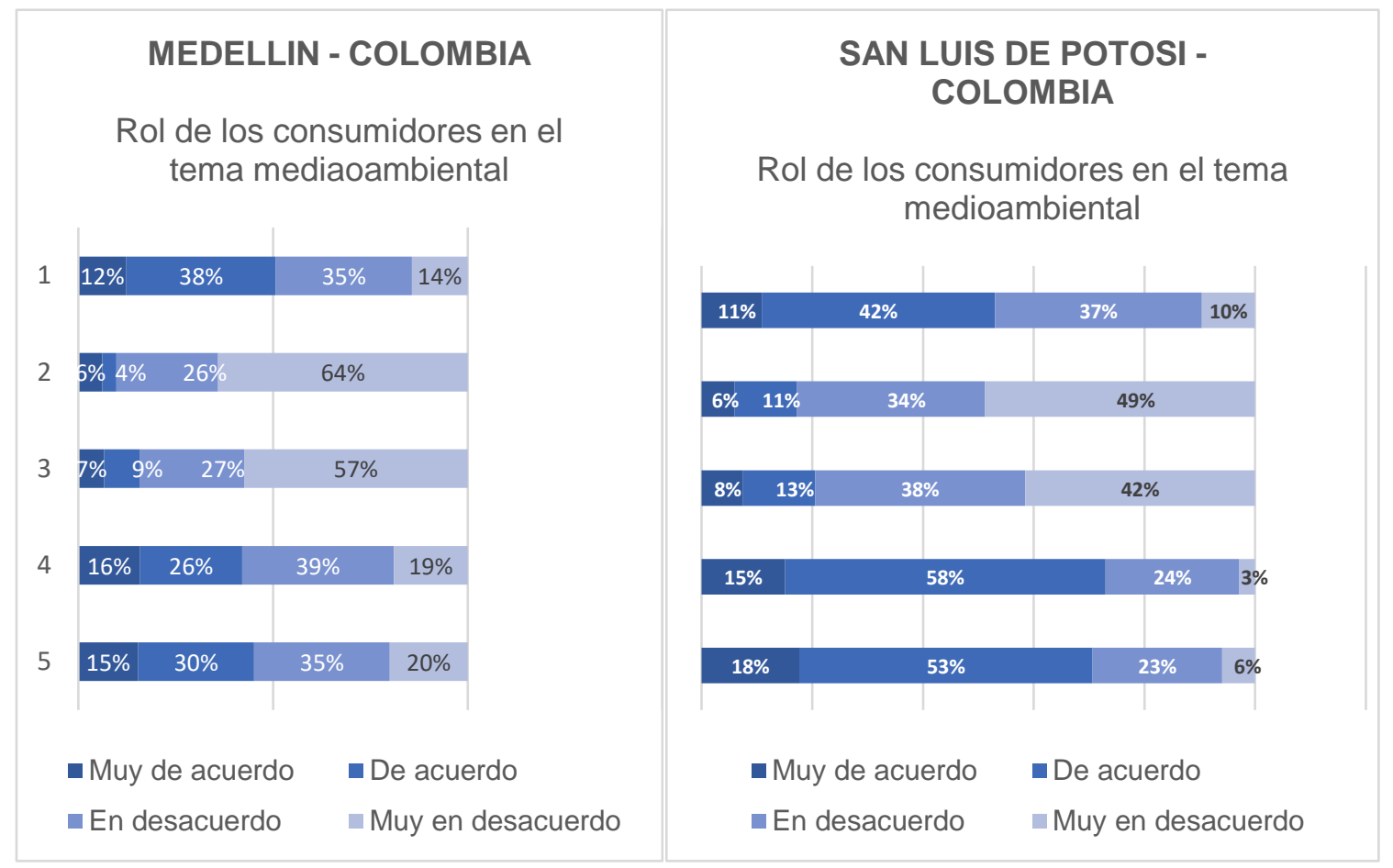

Fuente: Elaboración propia.

\begin{tabular}{|l|l|}
\hline 1 & $\begin{array}{l}\text { Aunque vea cosas que originan contaminación, no lo comunico a las autoridades que le compete } \\
\text { tomar medidas }\end{array}$ \\
\hline 2 & La contaminación medio ambiental no afecta personalmente mi vida \\
\hline 3 & No creo que la contaminación conduzca a los seres humanos a la ruina \\
\hline 4 & No quiero asistir a concentraciones relacionadas con la contaminación \\
\hline 5 & No deseo participar en protestas contra la contaminación \\
\hline
\end{tabular}




\section{DISCUSIÓN Y CONCLUSIONES:}

La investigación identificó una actitud o también llamada predisposición aprendida positiva, respecto a los temas medioambientales tanto para los consumidores de San Luis de Potosí como para Medellín Colombia.

Estos consumidores piensan que, el correcto manejo de los empaques es una de las formas de ayudar al cuidado medio ambiental, al momento de reutilizarlos o retornarlos ayudando en la economía circular, al igual que controlar el uso y el abuso del plástico; a tal punto, que están de acuerdo con que el gobierno adopte conductas coercitivas para sancionar a quienes no realicen estas prácticas.

Además, sobresale una actitud muy participativa y de apoyo en general a los temas medioambientales, considerando que es una temática que va más allá del consumo y que puede afectarle su vida a nivel personal; por tanto, tienen una actitud favorable respecto a todas las marchas, concentraciones y protestas que se desarrollen en pro de la conservación del medio ambiente.

Generar una actitud positiva es un paso muy importante en la construcción de una conducta, pero es muy claro el resultado de esta investigación, al mostrar que no está implícito el que esta actitud positiva de los consumidores conlleve una reacción a nivel conductual por parte del consumidor, lo cual se ve reflejado en que priman las variables; como pecio, calidad, entre otras, por encima de la consideración de las variables eco amigables 0 ambientales en el momento de la compra, siendo una conducta afianzada en los consumidores de San Luis de Potosí.
A nivel conductual pese a que las variables eco amigables no son las que tienen mayor peso en el momento de elegir un producto, si son de mayor consideración para los Consumidores de Medellín, al igual que el uso de empaques de tela para sus compras, lo cual permite concluir que los consumidores de Medellín no solamente tienen una actitud positiva a nivel medio ambiental, sino que ésta se ve reflejada en la consideración de conductas medio

ambientales como consumidores, respecto a los consumidores de San Luis de Potosí.

De acuerdo a estos hallazgos, ésta puede ser una de las respuestas al acelerado deterioro medioambiental como refleja la revisión de la literatura de esta investigación, puesto que no se logra llevar al consumidor de la actitud a la conducta, quizá se deban generar mayores estrategias que realmente impacten al consumidor, no solamente a nivel coercitivo, sino que ayuden a generar conciencia en el consumidor.

Es aquí donde el marketing juega un papel importante para lograr una sinergia entre las marcas (empresas) y el consumidor, para vincularlos en la cadena y que generen un trabajo de vía doble, generando conciencia del impacto de sus conductas en la preservación del medioambiente, como es el caso de Medellín donde la literatura referencia diversos estudios realizados alrededor del tema, porque existen planes gubernamentales, que como concluye el estudio, no solamente despierta la actitud en el consumidor sino lo lleva a la conducta. Este tipo de estrategias, aún no han sido implementadas en san Luis de Potosí, de acuerdo a la revisión de la literatura, lo cual hace que esta ciudad, se encuentre más lejos de desarrollar una conducta medioambiental, quedándose solamente en la actitud (intención); que hoy en día es una obligación ética y una tendencia en los países desarrollados (Webb et al., 2008), y finalmente, lleva a un elemento de transformación e impacto en la sociedad que 
depende de la conservación de los recursos naturales, pasando de un concepto antiguo de solo producción a un escenario de comportamientos colectivos para configurar un concepto más integral en un consumo socialmente responsable (CSR) (Pascual et al., 2015).

\section{REFERENCIAS:}

Duenas Ocampo, Sebastian, \& Perdomo-Ortiz, Jesús, \& Villa Castano, Lida Esperanza (2014). El concepto de consumo socialmente responsable y su medición. Una revisión de la literatura. Estudios Gerenciales, 30(132),287-300.[fecha de Consulta 4 de Septiembre de 2020]. ISSN: 0123-5923. Disponible en: https://www.redalyc.org/articulo. oa?id=212/21231380009

Elkington, J. y Hailes, J. (1989). The green consumer guide: From shampoo to champagne:

High-street shopping for a better environment. Londres: Gollancz.

González López, A. (2002). La preocupación por la calidad del medio ambiente. Un modelo cognitivo sobre la conducta ecológica. Tesis para optar al título de Doctor en Psicología social, Universidad Complutense de Madrid. Facultad de Psicología.

Madrid-España. Recuperado el 25 de abril de 2014, de http://biblioteca.ucm.es/tesis/psi/ ucm-t26479.pdf.

Holahan, C. (2005). Psicología ambiental, un enfoque general. México: Limusa Editores.
Kotler Philip y Armstrong Gary (2013). Fundamentos de Marketing. México. Décimo Primera Edición. Pearson Educación. Recuperado de: https://issuu.com/issuesoto/docs/fundamento s_de_marketing_-_philip_k

Maloney, M. P. y Ward, M. P. (1973). Ecology: Let's Hear from the People. American Psychologist, 583-586.

Mejia Gil, M.C., \& Puerta Silva C.P. (2018). Consumo Responsable y configuración de ciudadanías proambientales. Región \& Cohesión, 8 (1), 77-106.

Newhouse, N. (1990). Implications of attitude and behavior research for environmental conservation. Journal of Environmental Education, 22(1), 26- 36.

Organización de las Naciones Unidas, ONU. (1992). Declaración de Río sobre el Medio Ambiente y el Desarrollo: Agenda 21. Recuperado de: https://www.un.org/spanish/esa/sust dev/agenda21/index.htm

Pascual del Riquelme Martínez, M., Peñalosa Otero, M., \& López Célis, D. (2015). El consumo socialmente responsable en el mercado colombiano. Cuadernos Latinoamericanos Administración, 11(20), 61- $\quad 70$. https://doi.org/10.18270/cuaderlam.v11i20.62 4

Peñalosa Otero, Mónica Eugenia, \& López Celis, Diana María. (2018). El estereotipo de los colombianos frente al consumo socialmente responsable. Pensamiento \& Gestión, (44), 243260. https://dx.doi.org/10.14482/peg e.44.10384 
Shaw, D. y Shiu, E. (2002). An assessment of ethical obligation and self-identity inethical consumer decision-making: A structural equation modelling approach. International Journal of Consumer Studies, 26(4), 286-293.

Roth, Eric. (2000). Psicología ambiental: interfase entre conducta y naturaleza. Revista

Ciencia y Cultura, (8), 63-78. Recuperado en 04 de septiembre de 2020, http://www.scielo.org.bo/scielo.php? script=sci_arttext\&pid=S2077$33232000000200007 \&$ Ing=es\&tlng=

es.

Vargas Martínez, Elva Esther, \& López Moreda, Laureano José, \& Martínez Cervantes, Rosa Susana (2014). LA DIMENSIÓN AMBIENTAL EN LAS PREFERENCIAS DE

CONSUMO DEL TURISTA. CASO CANCÚN. Revista de Administração da Unimep, 12(2),42-58. ffecha de Consulta 4 de Septiembre de 2020]. ISSN: Disponible en: https://www.redalyc.org/articulo. oa?id=2737/273731798003

Webb, D. J., Mohr, L. A. y Harris, K. E. (2008). A reexamination of socially responsible consumption and its measurement. Journal of Business Research,61 (2), 91-98. 